and $\quad \frac{V}{\frac{3}{2} M}=\int_{0}^{u}\left(1-\frac{x^{3}}{\wp u-e_{1}}-\frac{\eta^{3}}{\wp u-e_{2}}-\frac{z^{2}}{\wp u-e_{3}}\right) d u$;

also $\int_{0}^{u} \frac{d u}{8 u-e_{\lambda}}=\frac{\zeta_{\lambda} u+e_{\lambda} u}{\left(e_{\lambda}-e_{\mu}\right)\left(e_{\nu}-e_{\lambda}\right)}$;

so that, if $a^{2}+\mu=\wp v-e_{1}, b^{2}+\mu=\wp v-e_{2}, c^{2}+\mu=\wp v v-e_{3}$ are the squares of the semi-axes of the confocal hyperboloid of one sheet, and if

$$
a^{2}+\nu=\wp w-e_{1}, \quad b^{2}+\nu=\wp w-e_{2}, \quad c^{2}+\nu=\wp w-e_{3},
$$

of the confocal hyperboloid of two sheets through the point ( $2 y z)$, then

$$
u=r \omega_{1}, \quad v=\omega_{1}+s \omega_{3}, \quad w=t \omega_{1}+\omega_{3},
$$

where $r, s, t$ are proper fractions; and

$$
x^{2}=\frac{\left(a^{2}+\lambda\right)\left(a^{2}+\mu\right)\left(a^{2}+v\right)}{\left(a^{2}-b^{3}\right)\left(a^{2}-c^{2}\right)}=\frac{\left(\frac{\sigma_{1} u}{\sigma u}\right)^{2}\left(\frac{\sigma_{1} v}{\sigma v}\right)^{2}\left(\frac{\sigma_{1} w}{\sigma w}\right)^{2}}{\left(e_{1}-e_{2}\right)\left(e_{1}-e_{3}\right)}
$$

with similar symmetrical expressions for $y^{2}$ and $z^{2}$.

On the Oonverse of Stereographic Projection and on Contangential and Coaxal Spherical Circles. By Mr. H. M. JEFFERY, F.R.S.

[Read May 13th, 1886.]

On Systems of Spherical Circles.

1. The first section is on a form of conical projection and introduces the equations and processes berein used. The second treats of systems of coaxal and contangential circles. Next, similitude and inversion are defined and illustrated. Lastly, the processes are applied to the solution of the problem of Contacts.

In developing the analogies to Plane Geometry, it is shown that theorems which are distinct in Planimetry are dual in Spherics; that those which relate to the magnitude of angles are identical in both Geometries; while theorems on arcs are modified when the radius of the sphere becomes infinite.

\title{
On the Converse of Stereographic Projection.
}

2. By stereographic projection, curves on a sphere are projected on an equatorial plane, whose pole is the pole of projection. The converse process is here considered; lines and curves on the equatorial 
or primitive plane are conically projected on the sphere from the same pole.

The two main familiar properties of this projection form the basis of the present investigation, (1) that all circles on the sphere are projected into circles or straight lines, constituting subcontrary sections of the cone of projection; (2) that an angle is unaltered by projection.

Spherical inversion and similitude, so far as spherical circles are concerned, are necessarily defined from this converse projection. ( $\$ 21$.) Since, in planimetry, an angle is unaltered by inversion, so in spherics it follows that an angle is also unaltered by spherical inversion, as is proved subsequently in $\S 24$.

3. To obtain the formuln of transformation by the converse of stereographio projection. (Fig. 1.)

Let $R(=1)$ be the radius of the sphere, of which $P^{\prime} Q P$ is a plane section, and $E^{\circ}$ the centre. $E D=r: P R$, its shadow on the sphere, $=\rho$; their mutual relation is

$$
r=\tan \frac{\rho}{2} .
$$

For

$$
\frac{r}{R}=\tan E P D=\tan \frac{\rho}{2}=\frac{\tan \rho}{1+\sec \rho} .
$$

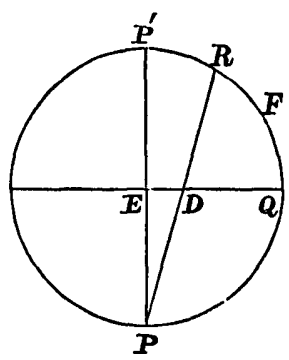

Fro. 1.

Also

$$
\frac{r^{2}}{\bar{R}^{2}}=\tan ^{2} \frac{\rho}{2}=\frac{\sec \rho-1}{\sec \rho+1} .
$$

The Cartesian coordinates $X(=r \cos \theta), Y(=r \sin \theta)$ are thus quadrically transformed into

$$
\frac{\tan \rho \cos \theta}{1+\sec \rho}, \frac{\tan \rho \sin \theta}{1+\sec \rho},
$$

or, in Gadermann's coordinate system and nomenclature,

$$
\frac{x}{1+\sqrt{ }\left(x^{2}+y^{2}+1\right)}, \frac{y}{1+\sqrt{ }\left(x^{3}+y^{2}+1\right)} .
$$

By $x, y, \rho$ he denotes $\tan x, \tan y, \tan \rho$, for brevity; thus,

$$
x=\tan \rho \cos \theta, \quad y=\tan \rho \sin \theta, x^{2}+y^{2}+1=\sec ^{2} \rho .
$$


4. To find the equation to a spherical circle referred to two tangent arcs as the axes of coordinates.

In plano, the equation to a circle referred to its two equal tangents $(c)$ as axes is $\quad r^{2}=x^{2}+y^{2}+2 x y \cos \omega=2 c(x+y)-c^{2}$;

when projected conically, as in $\S 3$, this becomes

$$
\frac{\sec \rho-1}{\sec \rho+1}-\frac{2 \tan t}{\sec t+1} \cdot \frac{x+y}{\sec \rho+1}+\frac{\sec t-1}{\sec t+1}=0 ;
$$

when simplified, $\quad \sec \rho=\cos t+(x+y) \sin t$

where

$$
\sec ^{2} \rho=1+x^{2}+y^{2}+2 x y \cos \omega,
$$

and $t$ is the tangent from $O$ the origin. This equation may be deduced from the general form referred to oblique axes (Gudermann's Sphärik,

$$
\sec \rho=\cos t(1+A x+B y) \text {. }
$$

By $x, y$ are denoted the tangents of the arcs intercepted on the quadrantal oblique axes $A O, B O$, by circles drawn through any point $(P)$ from the extremities $A, B$.

When $x=0, \rho=t=y$; when $y=0, \rho=t=a$; hence $A=B=\tan t$. This form (A) is useful for studying the properties of contangential circles.

5. If a secant drawn from a point meet a circle in two points, whose distances are $\rho_{1}, \rho_{2}$, and the polar of that point at a distance $R$,

$$
\cot \rho_{1}+\cot \rho_{2}=2 \cot R \text {. }
$$

The equation (A) may be written in the form

$$
\{\sin t-(x+y) \cos t\}^{2}=2 x y(1-\cos \omega) \text {. }
$$

As in plano, so in spherics thence derived by gnomonic projection,

$$
x \operatorname{cosec} \beta=y \operatorname{cosec} a=\tan \rho \operatorname{cosec} \omega ;
$$

if $\alpha, \beta$ denote the inclinations of the vector to the coordinate axes,

$$
\cot \rho_{1}+\cot \rho_{2}=2 \operatorname{cosec} \omega \cot t(\sin \alpha+\sin \beta)=2 \cot R \text {. }
$$

Cor.-By modifying the equation so as to include all sphero-conics,

$$
\{\sin t-(x+y) \cos t\}^{2}=m x y
$$

is applicable to them all, and is, in fact, the simplest case of Cotes' theorem adapted to Spherics. 
6. The tangential biangular equation to a circle, plane or spherical, referred to the ends of a chord (or radical axis) as poles, is the dual of the preceding form (A),

$$
\sec \rho=\cos c+(x+y) \sin c .
$$

In this coordinate system (Fig. 2) (explained Quarterly Math. Journal, Vol. xIII., p. 130), a moveable tangent line (or, in Spherics, a great circle) $T C P D$ meets two fixed lines (or great circles) perpendicular to $A B$ through its poles,

$$
\begin{aligned}
& \rho=D T A, \quad c=E A B=F B A, \\
& x=\tan D A B, \quad y=\tan C B A .
\end{aligned}
$$

In planimetry,

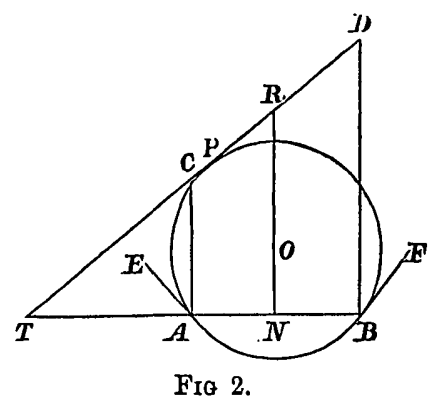

$$
\begin{aligned}
\cos c+(x+y) \sin c & =-\frac{O N}{r}+\frac{A B(A O+B D)}{2 r \times A B} \\
& =\frac{R O}{r}=\operatorname{cosec} T R N=\sec \rho .
\end{aligned}
$$

In spherics,

$$
\cos c+(x+y) \sin c=-\frac{\sin O N}{\sin r}+\frac{\tan A N(\tan A O+\tan B D)}{\tan r \sin A B} .
$$

The second term

$$
\begin{aligned}
= & \tan \rho \operatorname{cosec} A B \tan A N \cot r(\sin A T+\sin B T) \\
= & \tan \rho \cot r \sin T N \sec A N=\operatorname{cosec} r \tan R N \cos O N . \\
\text { Hence } \quad \cos c+(x+y) \sin c & =\operatorname{cosec} r \sin R O \sec R N \\
& =\operatorname{cosec} T R N \cdot \sec R N=\sec \rho .
\end{aligned}
$$

This equation is aseful for coaxal circles.

7. Dual theorem of $\S 5$. If from a point in a given line three other lines be drawn, two of them touching a conic, and the third through the pole of the given line with respect to the conic,

$$
\cot \rho_{1}+\cot \rho_{2}=2 \cot R,
$$

if $\rho_{1}, \rho_{2}, R$ denote the inclinations of the three lines to the given line. This theorem, which is equally applicable to Planimetry and Spherics, has been introdaced to illustrate the use of the equation (A). 
8. By this projection ( $(3)$ a plane $n^{\text {ic }}$ is generally projected into a spherical $(2 n)^{\text {lc }}$ with $n(n-1)$ nodes.

Let the quantic be arranged in order of its dimensions,

$$
u_{n}+u_{n-1}+\ldots+u_{0}=0 \text {. }
$$

When transformed, it becomes in spherical coordinates

$$
u_{n}+u_{n-1}(1+\sec \rho)+\ldots+u_{0}(1+\sec \rho)^{n}=0 .
$$

This may be written $\quad v_{n}+v_{n-1} \sec \rho=0$,

or, when rationalised, $\quad v_{n}^{2}=v_{n-1}^{2}\left(1+x^{2}+y^{2}\right)$.

The intersections of the curves $v_{n}, v_{n-1}$ are nodes.

Thus the projection of a conic (not being a circle) is a binodal spherical quartic.

9. The projections of a circle and of circular quantics may have lower dimensions.

Let the plane circle be

$$
x^{2}+y^{2}+2 a x+2 b y+c=0 .
$$

Its projection is the spherical circle

$$
(c+1) \sec \rho+2 a x+2 b y+c-1=0 .
$$

As is known, the centres of the two circles are not in the line of projection.

Let a circular cubic, of which a focus is at the origin, be written

$$
\left(x^{2}+y^{2}\right) u_{1}+v_{1}=0 \text {. }
$$

Its projection is the spherical binodal quartic with two double cyclic arcs, one of which is the quadrantal polar of the origin,

$$
\left(v_{1}-u_{1}\right)^{2}=\sec ^{2} \rho\left(v_{1}+u_{1}\right)^{2} \text {. }
$$

10. In transforming quadrically from plane to spherical tangential coordinates, conical projection is not directly applicable.

In Fig. 1 , let $P^{\prime} F$ be the complement of $P^{\prime} R$, or $p=\frac{\pi}{2}-\rho$.

Let also $p^{\prime}$ be the reciprocal of $r$ with respect to the sphere.

Then

$$
\tan \left(\frac{\pi}{4}-\frac{p}{2}\right)=\tan \frac{\rho}{2}=\frac{r}{R}=\frac{R}{p^{\prime}} .
$$


The following formulø of transformation are thus dual to those given in $\S 3$,

$$
\frac{R}{p^{\prime}}=\tan \left(\frac{\pi}{4}-\frac{p}{2}\right)=\frac{\cot p}{\operatorname{cosec} p+1}, \frac{R^{2}}{p^{2}}=\frac{\operatorname{cosec} p-1}{\operatorname{cosec} p+1} .
$$

Hence, if $R=1$, the Boothian coordinates,

$$
\xi\left(=\frac{1}{p^{\prime}} \cos \theta\right), \quad \eta\left(=\frac{1}{p^{\prime}} \sin \theta\right),
$$

become in Spherics

$$
\frac{\xi}{1+\operatorname{cosec} p}, \frac{\eta}{1+\operatorname{cosec} p}
$$

where

$$
\operatorname{cosec}^{8} p \doteq 1+\xi^{2}+\eta^{2} .
$$

To the reciprocal polar of the plane curve there corresponds, bat not by conical projection, the quadrantal polar of the projected plane curve.

Thus, the reciprocal polar of a circle is a conic, whose focus is at the origin; the corresponding spherical curves are both small circles, complementary to each other.

11. A plane class-cubic, which has a double focus at the origin, is transformed, as in $\S 10$, into a spherical class-quartic with two double foci, one of which is the origin, and two bitangents.

Usually, as in $\S 8$, to a plane class- $(n)^{10}$ there corresponds a spherical class- $(2 n)^{10}$ with $n(n-1)$ bitangents.

\section{On Contangential and Ooaxal Spherical Oircles.}

12. In Spherics, these systems of circles are dual or complementary to each other, i.e., a point in one circle is the pole of a tangent arc to its dual circle, their radii being comemplents of each other. In Spherics, a circle cannot beconsidered geometrically or analytically, without its twin antipodal circle, i.e., they must be treated together as the intersections of a sphere with a cone of twin-pair sheets, so that, whereas in plano there is but one real radical axis of two circles, the other being the line at infinity, in Spherics there are always two real radical axes of two circles, unless they are compolar or concentric. The radical axes of two small circles pass through the intersections of their compolar great circles. Hence it follows that the duals or quadrantal poles of the radical axes of two circles are the centres of similitude of the complementary circles, in which two pairs of common tangents intersect 
each other. The four radical centres of triads of circles, constituted by three small circles and their antipodal circles, are the duals or poles of the four axes of similitude of the corresponding triads of complementary circles. See infra, §§ 26, 27.

The two limiting point-circles in a coaxal system are the duals of limiting great circles of the complementary contangential system ; in the first coaxal system the radical axis is external, in the second the centre of similitude is internal.

13. The portions of the tangents, which are common to two small circles, which are terminated at the points of contact, are bisected in their radical axes; and the points of bisection are a quadrant apart.
13. The angle, under which two circles intersect each other, is bisected by the line joining either centre of similitude to a point of intersection; and the two centres of similitude subtend a right angle at such a point, real in Spherics, but imaginary in Planimetry.

If two circles be referred to their common tangents, as axes of coordinates, their equations are, by $\S 4$,

$$
\pm \sec \rho=\cos c+(x+y) \sin c, \quad \pm \sec \rho=\cos d+(x+y) \sin d .
$$

By combining them, we determine the two radical axes of the given circles and their antipodal circles,

$$
\cos c \pm \cos d+(x+y)(\sin c \pm \sin d)=0,
$$

or, separately, $\quad x+y-\tan \frac{1}{2}(c+d)=0$,

$$
x+y+\cot \frac{1}{2}(c+d)=0 .
$$

Remembering that $x, y$ denote $\tan x, \tan y$, we establish the two parts of the theorem, when $x=0, y=0$ separately.

14. In plano. When the radius of the sphere is infinite,

$$
x+y=\frac{1}{2}(c+d), \quad 1=0 ;
$$

the theorem holds good for a single radical axis (Gergonne, tom. viII., p. 323). For the dual theorem in plano, if the given circles intersect each other in two real points, as the forms of their equations imply, there is one, and but one, centre of similarity, and the second part of the theorem is inapplicable.

The next proposition is preliminary to the main property of radical axes.

VOL. XVII.-NO. 277. 
15. If from any point $P$ tangent arcs $t_{1}, t_{2}$ be drawn to two small circles, whose radii are $\delta_{1}$, $\delta_{2}$, and if perpendicular arcs $p_{1}, p_{2}$ be also drawn from $P$ on their radical axes,

$$
\begin{aligned}
& \cos ^{2} t_{1}-\cos ^{2} t_{2}=\Omega \sin p_{1} \sin p_{2}, \\
& \text { if } \Omega^{2}=\left(\sec ^{2} \delta_{1}-\sec ^{2} \delta_{2}\right)^{2} \\
& \quad+4 \sec ^{2} \delta_{1} \sec ^{2} \delta_{1} \sin ^{3} O_{1} O_{2} .
\end{aligned}
$$

In plano, $t_{1}^{2}-t_{2}^{2}=2 p\left(O_{1} O_{8}\right)$.
15. If a transversal intersect two small circles under angles $t_{1}, t_{2}$, and if perpendicular arcs $p_{1}, p_{2}$ be drawn from their centres of similitude on this great circle,

$$
\cos ^{3} t_{1}-\cos ^{2} t_{2}=\Omega \sin p_{1} \sin p_{9},
$$

if $\Omega^{2}=\left(\operatorname{cosec}^{2} \delta_{1}-\operatorname{cosec}^{3} \delta_{2}\right)^{2}$ $+4 \operatorname{cosec}^{2} \delta_{1} \operatorname{cosec}^{2} \delta_{2} \sin ^{2} O_{1} O_{2}$.

In plano, $\cos ^{2} t_{1}-\cos ^{2} t_{2}=p_{1} p_{2}\left(\frac{1}{\delta_{1}^{2}}-\frac{1}{\delta_{2}^{3}}\right)$.

If $\rho$ be the radius vector of $P(x, y),\left(h_{1}, k_{1}\right),\left(h_{2}, k_{2}\right)$ denote the centres $O_{1}, O_{2}$, and $T_{1}, T_{2}$ the tangents drawn from the origin to the circles,

$$
\begin{aligned}
\cos ^{2} t_{1}-\cos ^{2} t_{2} & =\sec ^{2} \delta_{1} \cos ^{2}\left(O_{1} P\right)-\sec ^{9} \delta_{9} \cos ^{2}\left(O_{2} P\right) \\
& =\cos ^{2} \rho\left\{\cos ^{2} T_{1}\left(1+x h_{1}+y k_{1}\right)^{2}-\cos ^{2} T_{2}\left(1+x h_{2}+y k_{2}\right)^{2}\right\}
\end{aligned}
$$

(Gudermann's Sphärik, §6; Graves' Appendix to Chasles' Spherical Conics, § 3)

$$
\begin{gathered}
=\cos ^{2} \rho\left\{\cos T_{1}-\cos T_{2}+x\left(h_{1} \cos T_{1}-h_{8} \cos T_{2}\right)\right. \\
\left.+y\left(k_{1} \cos T_{1}-k_{8} \cos T_{8}^{\prime}\right)\right\} \\
\times\left\{\cos T_{1}+\cos T_{2}+x\left(h_{1} \cos T_{1}+h_{2} \cos T_{2}\right)\right. \\
\left.+y\left(k_{1} \cos T_{1}+k_{2} \cos T_{2}\right)\right\} \\
\left.=\Omega_{1} \sin p_{1} \sin p_{2} \quad \text { (Gudermann, } \S 13 ; \text { Graves, } \S 4\right) .
\end{gathered}
$$

For $\left(\cos T_{1}-\cos T_{2}\right)^{2}+\left(h_{1} \cos T_{1}-h_{2} \cos T_{2}\right)^{2}+\left(k_{1} \cos T_{1}-k_{2} \cos T_{9}\right)^{2}$

$=\sec ^{3} r_{1} \cos ^{2} T_{1}+\sec ^{2} r_{2} \cos ^{2} T_{2}-2 \sec r_{1} \sec r_{2} \cos T_{1} \cos T_{2} \cos \left(O_{1} O_{2}\right)$

$=\sec ^{2} \delta_{1}+\sec ^{2} \delta_{2}-2 \sec \delta_{1} \sec \delta_{2} \cos \left(O_{1} O_{2}\right)$.

Cor. 1.-If tho product $\sin p_{1} \sin p_{2}$ be constant, i.e., if $P$ be a point in any splero-conic, which has the radical axes of the given circles for its cyclic arcs, the difference of the squares of the cosines of the tangent arcs ( $\left.\cos ^{2} t_{1}-\cos ^{2} t_{2}\right)$ is constant.

In plano the corresponding locus is a parallel to the radical axis.

Con. 2.-Conversely, if the difference $\left(\cos ^{2} t_{1}-\cos ^{2} t_{8}\right)$ has various constant values, the several loci of the point $P$ are biconcyclic spheroconics, whose cyclic arcs are the radical axes of the circles. 
Cok. 3.- If $X, Y, Z$ be three singly coaxal circles, the squares of the sines of the tangent arcs drawn from any point of one of them to the other two are in the ratio of multiples of the sines of the perpendiculars from that point on the separate radical axes ;

$$
\sin ^{2} t_{2} \operatorname{cosec} p_{2}: \sin ^{2} t_{8} \operatorname{cosec} p_{8}=\text { constant. }
$$

16. The following is a Geometrical proof of the dual theorem.

Let $P_{1}, P_{8}$ be the perpendicular arcs from $O_{1}, O_{2}$ on the transversal; $p_{1}, p_{2}$ those drawn from $Q_{1}, Q_{2} ; \delta_{1}, \delta_{2}$ the radii of the circles.

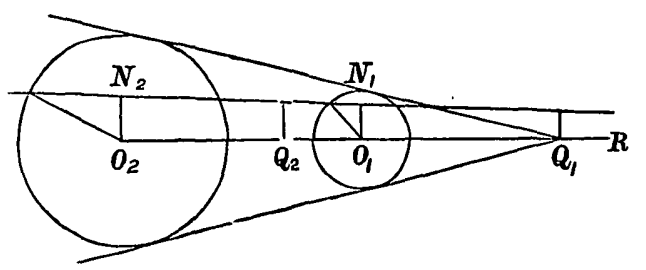

Fia. 3.

If $Q_{1} R$ be eliminated from the ratios, $\sin P_{1} \operatorname{cosec} O_{1} R=\sin P_{2} \operatorname{cosec} O_{2} R=\sin p_{1} \operatorname{cosec} Q_{1} R=\sin p_{2} \operatorname{cosec} Q_{2} R$, $\sin P_{1} \sin Q_{1} Q_{2}=\sin p_{2} \sin O_{1} Q_{1}+\sin p_{1} \sin O_{1} Q_{2}$, $\sin P_{2} \sin Q_{1} Q_{2}=\sin p_{2} \sin O_{2} Q_{1}-\sin p_{1} \sin O_{2} Q_{2}$.

Again, $\sin \delta_{1} \operatorname{cosec} O_{1} Q_{1}=\sin \delta_{2} \operatorname{cosec} O_{2} Q_{1}=\Omega_{1} \operatorname{cosec} O_{1} O_{2}$, $\sin \delta_{1} \operatorname{cosec} O_{1} Q_{2}=\sin \delta_{2} \operatorname{cosec} O_{2} Q_{2}=\Omega_{2} \operatorname{cosec} O_{1} O_{2}$,

if

$$
\begin{aligned}
& \Omega_{1}^{2}=\sin ^{2} \delta_{1}+\sin ^{2} \delta_{2}-2 \sin \delta_{1} \sin \delta_{2} \cos O_{1} O_{2}, \\
& \Omega_{2}^{2}=\sin ^{2} \delta_{1}+\sin ^{2} \delta_{2}+2 \sin \delta_{1} \sin \delta_{2} \cos O_{1} O_{2} .
\end{aligned}
$$

Hence $\quad \Omega_{1} \Omega_{2} \sin Q_{1} Q_{2}=2 \sin \delta_{1} \sin \delta_{2} \sin O_{1} O_{2}$.

For

$\frac{1}{\Omega_{1}} \sin \delta_{1}=\operatorname{cosec} O_{1} O_{2} \sin \left(O_{2} Q_{1}-O_{1} O_{2}\right)=\frac{1}{\Omega_{1}} \sin \delta_{2} \cos O_{1} O_{2}-\cos O_{2} Q_{1}$, $\frac{1}{\Omega_{2}} \sin \delta_{1}=\operatorname{cosec} O_{1} O_{2} \sin \left(O_{1} O_{2}-O_{2} Q_{2}\right)=\cos O_{2} Q_{2}-\frac{1}{\Omega_{2}} \sin \delta_{2} \cos O_{1} O_{2}$, $\Omega_{1} \Omega_{2} \sin Q_{1} Q_{2} \operatorname{cosec} O_{1} O_{2}$

$$
\begin{aligned}
& =\sin \delta_{2}\left(\sin \delta_{1}+\sin \delta_{2} \cos O_{1} O_{2}\right)-\sin \delta_{2}\left(\sin \delta_{2} \cos O_{1} O_{2}-\sin \delta_{1}\right) \\
& =2 \sin \delta_{1} \sin \delta_{2} .
\end{aligned}
$$


Consider the complements of the intersections $t_{1}, t_{2}$, and substitute the preceding values of $\sin P_{1}, \sin P_{2}$,

$$
\begin{aligned}
& \cos ^{2} t_{1}-\cos ^{2} t_{2}=\sin ^{2} P_{1} \operatorname{cosec}^{2} \delta_{1}-\sin ^{2} P_{8} \operatorname{cosec}^{2} \delta_{2} \\
&=\frac{4}{\Omega_{1} \Omega_{2}} \sin p_{1} \sin p_{2} \sin ^{2} O_{1} O_{2} \operatorname{cosec}^{2} Q_{1} Q_{2} \\
&=\Omega_{1} \Omega_{2} \sin p_{1} \sin p_{2} \operatorname{cosec}^{2} \delta_{1} \operatorname{cosec}^{2} \delta_{2} \\
&=\sin p_{1} \sin p_{2} \sqrt{ }\left\{\left(\operatorname{cosec}^{2} \delta_{1}+\operatorname{cosec}^{2} \delta_{2}\right)^{2}-4 \operatorname{cosec}^{2} \delta_{1} \operatorname{cosec}^{2} \delta_{2} \cos ^{2} O_{1} O_{2}\right\} .
\end{aligned}
$$

Hence the plane theorem may be deduced, or it may be proved independently.

Con. 1.-If the transversal be a tangent to a conic, spherical or plane, which has the centres of similitude for its foci, the difference of the squares of the cosines of the angles of intersection is constant.

Cor. 2.-If the transversal touch one of three singly contangential circles, the ratio $\frac{1}{p_{2}} \sin ^{2} t_{2}: \frac{1}{p_{3}} \sin ^{2} t_{8}$ is constant.

There cannot be three bi-contangential or three bi-coaxal circles.

17. To determine the limiting circles of a coaxal system.

They are definite or point-circles for the lowest limit, and the radical axis in all cases for the superior limit.

By taking the radical axis as the (y) axis of Gadermann's rectangular coordinates, any of the coaxal circles may be thus denoted in one or other of the two coazal systems :

$$
\text { (1) } \sec \rho \cos k=1+h x \text {, or (2) } \sec \rho=\cos k(1+h x) \text {, }
$$

according as the circles do or do not meet their radical axis.

In these equations, $h$ is the tangent of the abscissa of the centre; and $k$ in (1) either ordinate of two fixed points, or in (2) the tangent from the origin.

If $x, y$ denote, as usual, $\tan x, \tan y$,

$$
\sec ^{2} \rho=1+x^{2}+y^{2} \text {. }
$$

In the system (1), the limits are a small circle whose centre is the origin, and angular radius $k$, and the radical axis.

In the system (2), point-circles $( \pm k, 0)$ on the abscissa form the lowest limit.

In this case $h= \pm k, \quad \sec \rho=\cos k(1 \pm k x)$,

or, since $k$ denotes $\tan k$,

$$
y^{2}+(\sin k \pm x \cos k)^{2}=0 .
$$

The radical axis is the highest limit of the system. 
18. To determine the limiting circles of a contangential system of circles.

They are the centre of similitude and for the highest limit definite, small, or great circles.

The equations
(1) $\operatorname{cosec} p \sin \omega=1-h \xi$,
(2) $\operatorname{cosec} p=\cos t(1-h \xi)$,

which are the duals of those given in $\$ 17$, denote two contangential systems; in (1) the centre of similitude is external, in (2) internal, and the common tangents are imaginary.

In dualising, $x+\xi^{\prime}=0, y+\eta^{\prime}=0$, where $\xi^{\prime}, \eta^{\prime}$ denote the cotangents of the intercepts on the coordinate arcs of a line $\left(\xi^{\prime}, \eta^{\prime}\right)$, which is the quadrantal polar of the point $(x, y)$,

$$
\operatorname{cosec}^{2} p=1+\xi^{2}+\eta^{2} ;
$$

of the constants, $h$ ( $\equiv \tan h$ ) is unaltered with the centre.

In (1), $2 \omega=$ mutual inclination of a pair of common tangents, which meet in the exterior centre of similitude.

In (2), $\quad t=O P T=O P^{\prime} T^{\prime}$.

In Fig. 4, the circles are drawn in plano, which have $O$ for an internal centre of similitude; $T P$ is parallel to $T^{\prime} P^{\prime}$, and $O P P^{\prime}$ is perpendicular to the line of centres.

In Spherics, $O P P^{\prime}$ is the quadrantal polar of the origin, and $O$, the internal centre of similitude, is $90^{\circ}$ distant from the origin.

The superior limits of the system are, in

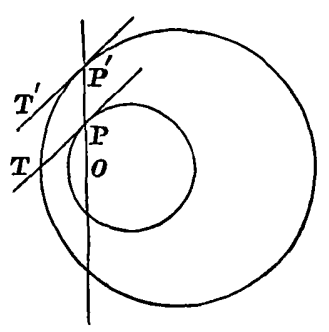

Fia. 4. (1), $p=\omega$, the small circle, whose centre is the origin and diameter $(2 \omega)$, and in (2), great circles,

$$
\left\{r^{2}+(\cos t \pm \xi \sin t)^{2}=0\right\}
$$

which are $(t)$ distant from the centre of similitude on either side.

In Plane Geometry, the limiting contangential circles are infinite, as also appears from considering Fig. 4 , since there is no finite limit to the system.

The inferior limits are the centres of similitude $(\xi=0)$.

19. Any circle which passes through the limiting point-circles of the coaxal system (2), and has its centre in their radical axis, is an orthogonal trajectors of that system.
19. Any small circle which touches the limiting great circles of the contangential system (2), and whose centre is equidistant from those great circles, has common tangents with circles of the system (2), quadrants in length. 
A circle which passes through the limiting point-circles of a coaxal system (2), and whose centre is in the $(y)$ axis of coordinates, belongs to the coaxal system (1),

$$
\sec \rho \cos k=1+l y \text {. }
$$

It cuts orthogonally any circle of the system (2),

$$
\sec \rho=\cos k(1+h x) \text {. }
$$

For the condition of coorthotomy is satisfied :

$$
\sec \delta_{1} \sec \delta_{9}=\sec k \sqrt{ }\left(1+l^{9}\right) \cos k \sqrt{ }\left(1+h^{9}\right)=\sec \left(O_{1} O_{9}\right),
$$

if $\delta_{1}, \delta_{2}$ are the radii, $O_{1}, O_{2}$ the centres of the two circles.

The following theorem is required for the next section.

20. If from any point a transversal be drawn to meet a fixed spherical circle,

$$
\tan \frac{\rho_{1}}{2} \tan \frac{\rho_{8}}{2}=\tan ^{2} \frac{t}{2},
$$

where $\rho_{1}, \rho_{9}$ denote the intercepts from that point, and $t$ the tangent arc, if the point is external. Hymers' Spherical Trig., p. 37.
20. If from any point in a line intersecting a fixed circle, plane or spherical, two tangents be drawn,

$$
\tan \frac{\rho_{1}}{2} \tan \frac{\rho_{9}}{2}=\tan ^{2} \frac{t}{2}
$$

where $\rho_{1}, \rho_{2}$ denote their inclinations to the initial line, and $t$ the inclination of a tangent at either intersection to that line.

This theorem may be deduced at once by conical projection from the plane property

$$
r_{1} r_{2}=t^{2}
$$

or it may be derived from the equation ( $\$ 4)$

$$
\sec \rho=\cos t+(x+y) \sin t .
$$

In the dual theorem, care must be taken to measure the angles $\rho_{1}, \rho_{9}$ in the same direction.

\section{On Similitude and Inversion.}

21. These relations are closely allied, since a centre of similitade is also a centre of inversion, for two circles.

If through a centre of similitude a vector arc be drawn to intersect two circles at
If from each point of a radical axis of two circles, plane or spherical, two tangents be drawn 
distances $\rho_{1}, \rho_{2}, \rho_{8}, \rho_{4}, \quad$ to them, the ratio of the tangents $\tan \frac{\rho_{1}}{2}: \tan \frac{\rho_{8}}{2}:: \tan \frac{\rho_{9}}{2}: \tan \frac{\rho_{4}}{2} \quad$ of half the angles, which they $\begin{array}{lll}c & d & \text { constant. (Chasles' Géométrie }\end{array}$ $:: \tan \frac{c}{2}: \tan \frac{d}{2} . \quad$ Supérieure, p. 468.)

(The tangent arcs are c, d.)

This theorem defines spherical similitude, and may be deduced by conical projection ( $\$ 3$ ) from the plane analogue.

By dualising, a second species of similitude is brought to light, in which the radical axis is also an axis of dual inversion.

A formal proof is obtained by the aid of $\S 4$.

22. Let the two circles be referred to their centre of similitude, not necessarily being external to both,

(1) $\sec \rho=\cos c+(x+y) \sin c, \quad$ (2) $\sec \rho=\cos d+(x+y) \sin d$,

if $c, d$ denote the intercepts on a common tangent arc.

For any transversal through the origin, both in Planimetry and Spherics, as is seen by gnomonic projection,

$$
x \operatorname{cosec} \beta=y \operatorname{cosec} \alpha=\rho \operatorname{cosec} 2 \omega,
$$

if $x, y, \rho$ in Spherics denote tangents, and $\alpha+\beta=2 \omega$.

Where it intersects (1),

$$
\begin{aligned}
\sec ^{2} \rho & =1+x^{2} \sin ^{2} 2 \omega \operatorname{cosec}^{2} \beta \\
& =\{\cos c+x(\sin \alpha+\sin \beta) \sin c \operatorname{cosec} \beta\}^{2} .
\end{aligned}
$$

Hence $\quad\{\sin \beta \sin c-x(\sin \alpha+\sin \beta) \cos c\}^{2}$

$$
\begin{aligned}
& =4 x^{2} \sin ^{2} \omega\left\{\cos ^{8} \frac{1}{2}(\alpha-\beta)-\cos ^{2} \frac{1}{2}(\alpha+\beta)\right\} \\
& =4 x^{2} \sin \alpha \sin \beta \sin ^{2} \omega .
\end{aligned}
$$

And $2 x \sin \omega\left\{\cos c \cos \frac{1}{2}(\alpha-\beta) \pm \sqrt{ }(\sin a \sin \beta)\right\}_{0}=\sin c \sin \beta$, $\sec \rho\left\{\cos c \cos \frac{1}{2}(\alpha-\beta) \pm \sqrt{ }(\sin \alpha \sin \beta)\right\}$

$$
=\cos \frac{1}{2}(\alpha-\beta) \pm \cos c \sqrt{ }(\sin \alpha \sin \beta) \text {. }
$$


If $\rho_{1}, \rho_{2}$ denote the vector arcs intercepted by the first circle,

$$
\begin{aligned}
\tan \frac{\rho_{1}}{2} & =\frac{\tan \rho_{1}}{1+\sec \rho_{1}}=\tan \frac{c}{2} \cos \omega\left\{\cos \frac{\alpha}{2}(\alpha-\beta)+\sqrt{ }(\sin \alpha \sin \beta)\right\} \\
& =\tan \frac{c}{2} \sec \omega\left\{\sqrt{ }\left(\cos \frac{a}{2} \cos \frac{\beta}{2}\right)-\sqrt{ }\left(\sin \frac{a}{2} \sin \frac{\beta}{2}\right)\right\}^{\prime}, \\
\tan \frac{\rho_{g}}{2} & =\tan \frac{c}{2} \sec \omega\left\{\sqrt{ }\left(\cos \frac{a}{2} \cos \frac{\beta}{2}\right)+\sqrt{ }\left(\sin \frac{a}{2} \sin \frac{\beta}{2}\right)\right\}^{\prime} .
\end{aligned}
$$

If $\rho_{9}, \rho_{4}$ denote the vector arcs for the second circle, they have similar values, so that

$$
\tan \frac{\rho_{1}}{2}: \tan \frac{\rho_{8}}{2}:: \tan \frac{\rho_{2}}{2}: \tan \frac{\rho_{4}}{2}:: \tan \frac{c}{2}: \tan \frac{d}{2} .
$$

CoR. 1. $\tan \frac{\rho_{1}}{2} \tan \frac{\rho_{g}}{2}=\tan ^{2} \frac{c}{2}$, as in $\S 20$.

CoR. 2. $\frac{V^{\prime}\left(\tan \frac{\rho_{8}}{2}\right)+\sqrt{ }\left(\tan \frac{\rho_{2}}{2}\right)}{\sqrt{ }\left(\tan \frac{\rho_{2}}{2}\right)-\sqrt{ }\left(\tan \frac{\rho_{1}}{2}\right)}=\sqrt{ }\left(\cot \frac{a}{2} \cot \frac{\beta}{2}\right)$.

CoR. 3. If $\tan ^{2} \frac{R}{2}=\tan \frac{c}{2} \tan \frac{d}{2}$, the circle, whose equation is

$$
\sec R=\cos \frac{1}{2}(c-d) \sec \frac{1}{2}(c+d),
$$

is coaxal with the two given circles, and has the common radical axis

$$
\dot{x}+y=\tan \frac{1}{2}(c+d) \text {. }
$$

This is also obvious geometrically, when the given circles intersect each other; for their circle of inversion must then pass through their intersections.

CoR. 4. Since

$$
\tan \frac{\rho_{1}}{2} \tan \frac{\rho_{4}}{2}=\tan \frac{\rho_{9}}{2} \tan \frac{\rho_{8}}{2}=\tan ^{2} \frac{R}{2},
$$

the centre of similitude is the centre of a system of points in involution, whose foci are on the circle of inversion.

23. On Inversion. - A centre of similitude of two contangential circles is also their centre
23. On Dual Inversion. - A radical axis of two circles is also an axis of dual inversion of those 
of inversion, and the circle $(R)$ circles, and is such that, if from of $\S 22$ is their circle of inversion. any point therein two pairs of tangents be drawn, making angles $\rho_{1}, \rho_{3} ; \rho_{3}, \rho_{4}$ with the axis, then in cross order

$$
\begin{aligned}
\tan \frac{\rho_{1}}{2} \tan \frac{\rho_{4}}{2} & =\tan \frac{\rho_{8}}{2} \tan \frac{\rho_{8}}{2} \\
& =\tan ^{2} \frac{R}{2} .
\end{aligned}
$$

The circle $(R)$ is compolar with the radical axis. See $\S 41$.

24. An angle is unaltered by inversion.

24. In Spherics, an aro of a great circle, and in Planimetry a straight line, is unaltered in length by Dual Inversion. See $\$ 41$.

In Planimetry an angle is unaltered by inversion; by conical projection, the same theorem is established in Spherics.

A formal proof is subjoined.

Let a pair of circles be written

$$
\text { (1) } c \sec r=1+m x+n y, \quad \text { (2) } c^{\prime} \sec r=1+m^{\prime} x+n^{\prime} y \text {. }
$$

The tangents at their points of intersection are

$$
\begin{aligned}
& c\left(1+x x^{\prime}+y y^{\prime}\right)=(1+m x+n y) \sec r^{\prime}, \\
& c^{\prime}\left(1+x x^{\prime}+y y^{\prime}\right)=\left(1+m^{\prime} x+n^{\prime} y\right) \sec r^{\prime} .
\end{aligned}
$$

If $\theta$ denote their mutual inclination, by Gudermann's Sphärik, § 11,

$$
\cos \theta=\frac{P}{Q R},
$$

$$
\text { where } \begin{aligned}
P= & \left(c x^{\prime}-m \sec r^{\prime}\right)\left(c^{\prime} x^{\prime}-m^{\prime} \sec r^{\prime}\right)+\left(c y^{\prime}-n \sec r^{\prime}\right)\left(c^{\prime} y^{\prime}-n^{\prime} \sec r^{\prime}\right) \\
& \quad+\left(c-\sec r^{\prime}\right)\left(c^{\prime}-\sec r^{\prime}\right) \\
= & c c^{\prime}\left(1+x^{\prime 2}+y^{\prime 2}\right)+\left(m m^{\prime}+n n^{\prime}+1\right) \sec ^{2} r^{\prime} \\
& \quad-\left[\left(m c^{\prime}+m^{\prime} c\right) x^{\prime}+\left(n c^{\prime}+r^{\prime} c\right) y^{\prime}+c+c^{\prime}\right] \sec r^{\prime} \\
= & \left(m m^{\prime}+n n^{\prime}-c c^{\prime}+1\right) \sec ^{2} r^{\prime}, \\
Q^{2}= & \left(c x^{\prime}-m \sec r^{\prime}\right)^{2}+\left(c y^{\prime}-n \sec r^{\prime}\right)^{2}+\left(c-\sec r^{\prime}\right)^{2} \\
= & \left(m^{2}+n^{2}-c^{2}+1\right) \sec ^{2} r^{\prime}, \\
R^{2}= & \left(m^{2}+n^{2}-c^{\prime 2}+1\right) \sec ^{2} r^{\prime} .
\end{aligned}
$$




\section{Hence}

$\left(m m^{\prime}+n n^{\prime}-c c^{\prime}+1\right) \sec \theta=\left(m^{2}+n^{4}-c^{2}+1\right)^{\prime}\left(m^{2}+n^{2}-c^{2}+1\right)^{\prime}$.

Next, to obtain the inverse circles.

Write (1) in the form

$$
c\left(1+\tan ^{2} \frac{r}{2}\right)=1-\tan ^{2} \frac{r}{2}+2(m \cos \theta+n \sin \theta) \tan \frac{r}{2} .
$$

Its inverse is, if

$$
\tan \frac{r}{2} \tan \frac{\rho}{2}=\kappa,
$$

$$
\sec \rho\left\{\kappa^{2}(c+1)+c-1\right\}=c-1-\kappa^{2}(c+1)+2 \kappa(m x+n y) .
$$

The inverse of (2) is

$$
\sec \rho\left\{\kappa^{2}\left(c^{\prime}+1\right)+c^{\prime}-1\right\}=c^{\prime}-1-\kappa^{2}\left(c^{\prime}+1\right)+2 \kappa\left(m^{\prime} x+n^{\prime} y\right) .
$$

The fanction $P$ becomes for these values

$$
\begin{aligned}
4 \kappa^{2}\left(m m^{\prime}+n n^{\prime}\right) & +\left\{(c-1)-\kappa^{2}(c+1)\right\}\left\{\left(c^{\prime}-1\right)-\kappa^{2}\left(c^{\prime}+1\right)\right\} \\
& -\left\{(c-1)+\kappa^{2}(c+1)\right\}\left\{\left(c^{\prime}-1\right)+\kappa^{9}\left(c^{\prime}+1\right)\right\},
\end{aligned}
$$

or $\quad 4 \kappa^{2}\left(m m^{\prime}+n n^{\prime}-c c^{\prime}+1\right)$.

Similarly, $\quad Q^{2}$ becomes $4 \kappa^{2}\left(m^{2}+n^{2}-c^{2}+1\right)$,

$$
R^{2} \quad \text { " } 4 \kappa^{2}\left(m^{2}+n^{2}-c^{2}+1\right) \text {. }
$$

The angle of intersection $(\theta)$ is therefore unaltered by inversion.

25. If through a centre of similitude any two arcs be drawn meeting the first circle in the points $R, R^{\prime}, S, S^{\prime}$, and the second in the points $\rho, \rho^{\prime}, \sigma, \sigma^{\prime}$, then the $\operatorname{arcs} R S, \rho \sigma ; R^{\prime} S^{\prime}, \rho^{\prime} \sigma^{\prime}$ will meet on one radical axis; and the $\operatorname{arcs} R S, \rho^{\prime} \sigma^{\prime} ; R^{\prime} S^{\prime}, \rho \sigma$ will meet on the other radical axis of the two circles.

The two quadrilaterals $R R^{\prime} S S^{\prime}, \rho \rho^{\prime} \sigma \sigma^{\prime}$ have $O$ for a common centre, and the other two pairs of centres are collinear with $O$, the lines of collinearity forming a harmonic pencil with the other collinear sides ORp, OSo. See Fig. in Salmon's Conic Sections, p. 107.
25. If from any two points in a radical axis two pairs of tangent arcs be drawn, so as to constitute two quadrilaterals with a common diagonal arc,-the arcs which join the angular points, one taken from each quadrilateral, will pass through one or other centre of similitude. 
As in $\S 22$, let the two circles be referred to the common tangents

(1) $\sec \rho=\cos c+(x+y) \sin c$,

(2) $\sec \rho=\cos d+(x+y) \sin d$.

For any transversal $O \rho R$,

$$
x \operatorname{cosec} \beta=y \operatorname{cosec} \alpha=\rho \operatorname{cosec} 2 \omega,
$$

where it intersects (1), as in $\S 22$,

$$
\frac{1}{\rho} \sin c \cos \omega=\cos c \cos \frac{1}{2}(\alpha-\beta) \pm \sqrt{ }(\sin \alpha \sin \beta) ;
$$

the two values of $\rho$ denote $\tan O \rho^{\prime}, \tan O \rho$.

So also are denoted $\tan O R^{\prime}, \tan O R$, when $d$ is written for $c$.

For a second transversal $0 \sigma S$,

$$
\begin{gathered}
x \operatorname{cosec} \delta=y \operatorname{cosec} \gamma=\rho \operatorname{cosec} 2 \omega ; \\
\frac{1}{\rho^{\prime}} \sin c \cos \omega=\cos c \cos \frac{1}{2}(\gamma-\delta) \pm \sqrt{ }(\sin \gamma \sin \delta),
\end{gathered}
$$

whose two values denote $\tan O \sigma^{\prime}, \tan O \sigma$. Similarly, we express $\tan O S^{\prime}, \tan O S$.

The equation to the ohord $\rho^{\prime} \sigma^{\prime}$ is

$$
\begin{array}{r}
\left(x-\rho_{1} \sin \beta \operatorname{cosec} 2 \omega\right)\left(\rho_{1} \sin \alpha-\rho_{1}^{\prime} \sin \gamma\right) \\
=\left(y-\rho_{1} \sin \alpha \operatorname{cosec} 2 \omega\right)\left(\rho_{1} \sin \beta-\rho_{1}^{\prime} \sin \delta\right), \\
\text { or } x\left(\frac{1}{\rho_{1}^{\prime}} \sin a-\frac{1}{\rho_{1}} \sin \gamma\right)-y\left(\frac{1}{\rho_{1}^{\prime}} \sin \beta-\frac{1}{\rho_{2}} \sin \delta\right) \\
=(\sin u \sin \delta-\sin \beta \sin \gamma) \operatorname{cosec} 2 \omega .
\end{array}
$$

The equation to the chord RS is

$$
\begin{aligned}
x\left(\frac{1}{R_{2}^{\prime}} \sin \alpha-\frac{1}{R_{8}} \sin \gamma\right) & -y\left(\frac{1}{R_{2}^{\prime}} \sin \beta-\frac{1}{R_{g}} \sin \delta\right) \\
= & (\sin \alpha \sin \delta-\sin \beta \sin \gamma) \operatorname{cosec} 2 \omega .
\end{aligned}
$$

Multiply (3) by $\sin c,(4)$ by $\sin d$, and add :

$$
\begin{aligned}
& \sec \omega(\cos c+\cos d)\left\{x\left[\sin \alpha \cos \frac{1}{2}(\gamma-\delta)-\sin \gamma \cos \frac{1}{2}(\alpha-\beta)\right]\right. \\
&\left.-y\left[\sin \beta \cos \frac{1}{2}(\gamma-\delta)-\sin \delta \cos \frac{1}{2}(\alpha-\beta)\right]\right\} \\
&=\operatorname{cosec} 2 \omega(\sin c+\sin d)(\sin \alpha \sin \delta-\sin \beta \sin \gamma) \ldots \ldots \ldots
\end{aligned}
$$


But, since $\alpha+\beta=\gamma+\delta=2 \omega$, the coefficient of $x$ is

$$
\sin \alpha \cos (\gamma-\omega)-\sin \gamma \cos (\alpha-\omega)=\sin (\alpha-\gamma) \cos \omega,
$$

and $\sin \alpha \sin (2 \omega-\gamma)-\sin \gamma \sin (2 \omega-\alpha)=\sin 2 \omega \sin (a-\gamma)$.

After these and like reductions, (5) takes the form of the radical axis, in which (3) and (4) meet (see $\S 13$ ),

$$
x+y=\tan \frac{1}{a} \cdot(c+d)
$$

Similarly, it may be shown that $\rho \sigma$ and $R^{\prime} S^{\prime}$ intersect in (5).

The equation to $R^{\prime} S^{\prime}$ is

$$
\begin{aligned}
x\left(\frac{1}{R_{1}^{\prime}} \sin a-\frac{1}{R_{1}} \sin \gamma\right) & -y\left(\frac{1}{R_{1}^{\prime}} \sin \beta-\frac{1}{R_{1}} \sin \delta\right) \\
= & (\sin a \sin \delta-\sin \beta \sin \gamma) \operatorname{cosec} 2 \omega .
\end{aligned}
$$

Maltiply (3) by $\sin c,(6)$ by $\sin \delta$, and subtract one from the other.

The resulting equation may be reduced, as (5) was, to denote the second radical axis (see $§ 13$ ),

$$
x+y+\cot \frac{1}{2}(c+d)=0
$$

Similarly, it may be shown that $\rho^{\prime} \sigma^{\prime}$ and $R S$ meet in (7).

The centres of the two quadrilaterals lie in the two lines

$$
x \sqrt{ }(\sin \alpha \sin \gamma) \pm y \sqrt{ }(\sin \beta \sin \delta)=0 .
$$

CoR.-The tangents at $R, \rho$; $R, \rho^{\prime}$ meet on one radical axis ; those at $R, \rho^{\prime} ; R, \rho$ meet on the other radical axis.
Cor.-Arcs which join the points of contact of a pair of tangents, which are drawn from any point in the radical axis to one circle, to the points of contact of a pair of tangents to the second. circle, intersect in one or other centr' $\theta$ of similitide.

\section{On the Problem of Oontacts.}

26. To describe pairs of spherical circles, which shall touch three given circles and their antipodal circles, by triads.

Let the radical centre of the three given circles be taken as the origin of Gadermann's coordinates; their equations may be written

$$
\left.\begin{array}{l}
\sec t \sec \rho=1+a x+b y \\
\sec t \sec \rho=1+c x+d y \\
\sec t \sec \rho=1+e x+f y
\end{array}\right\}
$$


The centres or poles are $(a, b),(c, d),(e, f)$, severally; $t$ is the tangent arc drawn from the origin to any of the three circles, supposed to be external to each other (see Fig. in Salmon's Conics, p. 112).

Also $\sec ^{3} \rho=1+x^{2}+y^{2}$, if $x, y, a, b, \ldots$ denote the tangents of those arcs, as usual. The Jacobian of the three circles,

$$
\sec \rho=\sec t,
$$

cuts them orthogonally, since it satisfies the test of coorthotomy (Proceedings, Vol. xVI., p. 111).

The centre of the Jacobian is also the centre of similitude of a required pair of circumscribed circles (Salmon, loc. cit.), and therefore the Jacobian is their circle of inversion, and therefore also coaxal with them, their common radical axis being the axis of similitude of the three given circles. (Casey, Sequel to Euclid, p. 118.)

There are four radical centres, including the origin,

$$
a x+b y+1= \pm(c x+d y+1)= \pm(e x+f y+1) ;
$$

but at present only the positive signs are considered. And there are four corresponding Jacobian orthotomic circles, of which that which cuts the three given circles is

$$
\sec \rho=\sec t .
$$

The equivalent Boothian tangential equations to the given circles have the form

$$
\sin ^{2} r_{1}\left(a^{2}+b^{2}+1\right)\left(\xi^{2}+\eta^{2}+1\right)=(a \xi+b \eta-1)^{2},
$$

or, since

$$
a^{2}+b^{2}+1=\sec ^{2} t \sec ^{2} r_{1} \text {, }
$$

$$
\tan ^{2} r_{1} \sec ^{2} t\left(\xi^{2}+\eta^{2}+1\right)=(a \xi+b \eta-1)^{2} \text {. }
$$

There are therefore four axes of similitude, thus denoted

$$
\begin{aligned}
& \pm \theta \sec t \tan r_{1}+a \xi^{\prime}+b \eta^{\prime}-1=0, \\
& \pm \theta \sec t \tan r_{2}+c \xi^{\prime}+d \eta^{\prime}-1=0, \\
& \pm \theta \sec t \tan r_{3}+e \xi^{\prime}+f \eta^{\prime}-1=0 .
\end{aligned}
$$

The set of values here required is found by giving the same sign to $\tan r_{1}, \tan r_{2}, \tan r_{3}$,

$$
\frac{\theta \sec t}{|a, d, 1|}=\frac{-\xi^{\prime}}{\left|\tan r_{1}, d, 1\right|}=\frac{\eta^{\prime}}{\left|\tan r_{1}, c, 1\right|}=\frac{1}{\left|\tan r_{1}, c, f\right|} .
$$

By the preceding theorems, the required equation to a pair of cir- 
cumscribed circles must bave the form

$$
\sec \rho=\sec t+\mu\left(x \xi^{\prime}+y \eta^{\prime}-1\right) .
$$

But it is better to introduce first another circle coorthotomic with the Jacobian, and having the axis of similitude $\left(\xi^{\prime}, \eta^{\prime}\right)$ for their radical axis,

$$
\sec \rho=\cos t+\sin t \tan t\left(x \xi^{\prime}+y \eta^{\prime}\right) .
$$

We will now thus present the required pair of circles,

$$
(1+r) \sec \rho=\cos t+r \sec t+\sin t \tan t\left(x \xi^{\prime}+y \eta^{\prime}\right)
$$

The multiplier $(r)$ must be found from the condition that the pair touch each of the three given circles.

In general, for the condition of contact for two circles, whose spherical radii are $r_{1}, R$, and whose equations are

$$
\begin{gathered}
\cos r_{1} \sec \rho\left(1+a^{2}+b^{2}\right)^{i}=1+a x+b y, \\
\cos R \sec \rho\left(1+a^{2}+\beta^{2}\right)^{1}=1+a x+\beta y, \\
\cos \left(r_{1} \sim R\right)=\cos D=(1+a a+b \beta)\left(1+a^{2}+b^{2}\right)^{-1}\left(1+a^{2}+\beta^{2}\right)^{-1} .
\end{gathered}
$$

Let these conditions be applied to (2) and the first circle of (1).

In this case

$$
\begin{gathered}
a^{2}+b^{2}+1=\sec ^{2} t \sec ^{2} r_{1}, \\
\left(1+a^{2}+\beta^{2}\right)^{1}(\cos t+r \sec t)=(1+\tau) \sec R .
\end{gathered}
$$

With these values, the condition of contact becomes

$$
\begin{aligned}
(1+\tau) \tan r_{1} \tan R & =\left(a \xi^{\prime}+b \eta^{\prime}-1\right) \sin ^{2} t \\
& =-\theta \sin ^{2} t \sec t \tan r_{1}
\end{aligned}
$$

Eliminate $R$ from (3) and (4),

$$
(1+\tau)^{2}+\theta^{2} \sin ^{2} t \tan ^{2} t=(\cos t+\tau \sec t)^{2}+\left(\xi^{2}+\eta^{2}\right) \sin ^{2} t \tan ^{2} t .
$$

Hence, finally, the two values of $\tau$ in (2) are obtained in terms of known constants, $\quad \tau^{2}=\cos ^{2} t-\left(\xi^{2}+\eta^{2}-\theta^{2}\right) \sin ^{2} t$.

This equation (2) and the values of $r$ have been otherwise obtained by Gergonne's process (Salmon's Conics, p. 110).

But since different radical centres (other than the origin) and Jacobians are necessary for the other cases, where one or more of the antipodal circles are concerned, three-point coordinates are preferable to exhibit the equations collectively.

27. To find the equations to pairs of spherical circles, which shall 
touch three given small circles, and their antipodal circles, when three point-coordinates are employed.

Let $A B O$, the triangle of reference, have the centres of the given circles for its angular points; if $a, b, c ; a, \beta, \gamma$ denote the sines of the sides and the perpendicular coordinates, and if $r_{1}, r_{2}, r_{3}$ be the angular radii, and $6 V=b c \sin A$ (in Todhunter's Spherical Trig., p. 22, $n=3 V)$. For the given circles and their antipodal circles,

$$
\left.\begin{array}{l}
S_{1} \equiv a a+b \beta \cos c+c \gamma \cos b \mp 6 V \cos r_{1}=0 \\
S_{2} \equiv a a \cos c+b \beta+c \gamma \cos a \mp 6 V \cos r_{2}=0 \\
S_{8} \equiv a \alpha \cos b+b \beta \cos a+c \gamma \mp 6 V \cos r_{8}=0
\end{array}\right\}
$$

The equations are rendered homogeneous by the fandamental relation (see Salmon's Solid Geometry, Sphero-Conics, p. 198, where threepoint and three-line coordinates are introduced),

$$
\Sigma\left(a^{2} a^{2}+2 b c \beta \gamma \cos a\right)=(6 V)^{2} .
$$

The given circles have four radical centres, which are also the centres of the corresponding Jacobians,

$$
\begin{aligned}
(a \alpha+b \beta \cos c+c \gamma \cos b) \text { sec } r_{1} & = \pm(a \alpha \cos c+b \beta+c \gamma \cos a) \sec r_{8} \\
& = \pm(a \alpha \cos \ddot{b}+b \beta \cos a+c \gamma) \sec r_{8} .
\end{aligned}
$$

The Jacobians of the several triads of circles are

$$
\begin{gathered}
\pm a a \cos r_{1} \pm b \beta \cos r_{2} \pm c \gamma \cos r_{s}=6 V, \\
a a S_{1}+b \beta S_{2}+c \gamma S_{8}=0 .
\end{gathered}
$$

or

The Jacobians intersect the several triads orthogonally, for it was shown (Proceedings, Vol. xvi., p. 111) that the condition of coorthotomy for two circles, $\left[l \alpha+m \beta+n \gamma=d, l^{\prime} \boldsymbol{\alpha}+m^{\prime} \beta+n^{\prime} \gamma=d^{\prime}\right]$, is

$$
\Sigma\left\{l l^{\prime}-\left(m^{\prime} n+m n^{\prime}\right) \cos A\right\}=d d^{\prime} \text {. }
$$

These conditions are satisfied by the Jacobians.

The tangential equivalents to the circles (1) are

$$
p^{2}=r_{1}^{2} ; \quad q^{2}=r_{2}^{2} ; \quad r^{2}=r_{8}^{2} ;
$$

if $p, q, r$ denote the sines of the perpendiculars drawn from $A, B, C$ on a tangent arc. For the four axes of similitude,

$$
\frac{p^{2}}{r_{1}^{2}}=\frac{q^{2}}{r_{g}^{2}}=\frac{r^{3}}{r_{s}^{2}}
$$

or, in point-coordinates, $a a r_{1} \pm b \beta r_{2} \pm c \gamma r_{s}=0$, also $r_{1}, r_{2}, r_{3}$ now denote $\sin r_{1}, \ldots$ 
As has been stated in $\$ 26$, the required equation to a pair of circumscribed circles is coaxal with a Jacobian, the radical axis being the axis of similitude of the corresponding triad of circles,

$a a\left(\cos r_{1}+\tau \sin r_{1}\right)+b \beta\left(\cos r_{2}+r \sin r_{3}\right)+c \gamma\left(\cos r_{3}+\tau \sin r_{3}\right)=6 V \ldots$ (3).

The two values of the multiplier $(r)$ are found by the condition that this circle (3) touches any one of the circles (1).

In general, if $\rho_{1}, \rho_{2}$ be the angular radii of two touching circles, and $D$ be the connector of their centres,

$$
\begin{gathered}
\cos \left(\rho_{1} \pm \rho_{8}\right)=\cos D, \\
\text { or } \quad\left(\cos \rho_{1} \cos \rho_{2}-\cos D\right)^{2}=\left(1-\cos ^{2} \rho_{1}\right)\left(1-\cos ^{8} \rho_{2}\right) .
\end{gathered}
$$

If, then, their equations be

$$
l a+m \beta+n \gamma=d, \quad l^{\prime} \alpha+m^{\prime} \beta+n^{\prime} \gamma=d^{\prime},
$$

the necessary condition for contact is

$$
\begin{gathered}
\left\{\Sigma\left[l l^{\prime}-\left(m n^{\prime}+m^{\prime} n\right) \cos A\right]-d d^{\prime}\right\}^{3} \\
=\left\{\Sigma\left(l^{2}-2 m n \cos A\right)-d^{2}\right\}\left\{\Sigma\left(l^{\prime 2}-2 m^{\prime} n^{\prime} \cos A\right)-d^{\prime y}\right\} .
\end{gathered}
$$

If this condition be applied in this case,

$$
\begin{gathered}
\left(1+\tau^{2}\right)(6 \nabla)^{2} \\
=\Sigma\left\{a^{2}\left(\cos r_{1}+\tau \sin r_{1}\right)^{2}-2 b c\left(\cos r_{2}+\tau \sin r_{8}\right)\left(\cos r_{3}+\tau \sin r_{3}\right) \cos A\right\} .
\end{gathered}
$$

Since there are four radical centres, and four axes of similitude, there are in all 16 pairs of circumscribed circles, or 32 solutions in all.

Cor. 1. If $r_{1}=r_{2}=r_{8}=0$, there is no axis of similitude, and the Jacobians denote the four circles described about $A B C$ and its associated triangles, $A^{\prime} B C, A B^{\prime} C, A B C^{\prime}$, whose equations are

$$
\pm a \alpha \pm b \beta \pm c \gamma=6 \mathrm{~V} \text {. }
$$

Cor. 2. If $r_{1}=r_{2}=r_{3}=\frac{1}{2} \pi$, the Jacobian is the imaginary circle $(6 V=0), r^{2}=\cot ^{2} h$, if $R$ be the radius of circumscribed circle; and the equation is derived to the circle inscribed in the polar triangle of $A B C$,

$$
a a+b \beta+c \gamma=6 \nabla \tan R,
$$

whose angular radins is therefore the complement of $R$. (Todhunter's Spher. Trig., p. 66.)

28. Dr. Casey has pointed out that each pair of circles circumscribed about a triad of circles is a degenerate case of spherocyclides, when the dirigent conic has double contact with the Jacobian, 
In the Proceedings of the Royal Irish Academy, Vol. Ix., p. 396, he obtained the equation to such a pair of circles,

$$
\sin \frac{l}{2} \sqrt{ }\left(S_{1} \sec r_{1}\right)+\sin \frac{m}{2} \sqrt{ }\left(S_{2} \sec r_{2}\right)+\sin \frac{n}{2} \sqrt{ }\left(S_{8} \sec r_{8}\right)=0,
$$

where $l, m, n$ denote the lengths of tangent arcs common to pairs of the given triad of circles, and $S_{1}, S_{3}, S_{s}$ denote their equations given in $\$ 27$.

I propose to obtain Dr. Casey's equation both analytically and geometrically.

29. In general, if the variable circle, which generates a spherocyclide, be $\quad a a L+b \beta M+c \gamma N=6 \mathrm{~V}$

and the Jacobian of three given circles be, as in $\S 27$,

$$
a \alpha \cos r_{1}+b \beta \cos r_{2}+c \gamma \cos r_{8}=6 V
$$

then, if the pole of (1), which moves so as to intersect (2) orthogonally, be made to lie on the dirigent conic

$$
\left(u, v, w, u i^{\prime}, v^{\prime}, w^{\prime} \gamma p, q, r\right)^{2}=0,
$$

the sphero-cyclide will be denoted by the equation

$$
\left(u, v ; w, u^{\prime}, v^{\prime}, w^{\prime} \gamma S_{1}, S_{2}, S_{3}\right)^{2}=0 .
$$

For, by a known theorem of Dr. Casey, quadric transformation is thus effected from the tangential coordinates

$$
p: q: r:: S_{1}: S_{2}: S_{8} \text {. }
$$

(Casey on Cyclides, $\S 40$. See also a proof in Proceedings, Vol. XVI., p. 115).

30. To determine the dirigent conic, when it has double contact with the Jacobian.

Let the given triad of circles (1), in $\$ 27$, be supposed to intersect each other at the angles $\phi_{1}, \phi_{2}, \phi_{3}$; then, if $\left(\widetilde{S_{2}, S_{3}}\right)=\phi_{1}$,

$$
\begin{aligned}
\cos a & =\cos r_{2} \cos r_{3}+\sin r_{2} \sin r_{8} \cos \left(\pi-\phi_{1}\right) \\
& =\cos \left(r_{2}-r_{3}\right)-2 \sin r_{2} \sin r_{8} \cos ^{2} \frac{\phi_{1}}{2} .
\end{aligned}
$$

Next, let $l, m, n$ denote, as in $\S 28$, common tangent arcs,

$$
\begin{aligned}
\cos a & =\sin r_{2} \sin r_{3}+\cos r_{2} \cos r_{8} \cos l \\
& =\cos \left(r_{2}-r_{3}\right)-2 \cos r_{2} \cos r_{3} \sin ^{2} \frac{l}{2} .
\end{aligned}
$$

voL. XVII.-NO. 278. 
Now, remembering that $(6 V)^{2}=\Sigma\left(a^{2} a^{3}+2 b c \beta \gamma \cos a\right)$,

the Jacobian may be written

$$
a^{2} a^{2} \sin ^{2} r_{1}+b^{2} \beta^{3} \sin ^{2} r_{2}+\ldots-2 b c \beta \gamma \sin r_{2} \sin r_{3} \cos \phi_{1}-\ldots=0,
$$

or $\quad\left(a a \sin r_{1}+b \beta \sin r_{2}+c \gamma \sin r_{s}\right)^{2}=4 b c \beta \gamma \sin r_{2} \sin r_{s} \cos ^{2} \frac{\phi_{1}}{2}+\ldots \ldots$.

Since the dirigent conic has double contact with the Jacobian, its form must be

$$
\frac{1}{u \alpha} \operatorname{cosec} r_{1} \cos ^{2} \frac{\phi_{1}}{2}+\frac{1}{b \beta} \operatorname{cosec} r_{2} \cos ^{2} \frac{\phi}{2}+\frac{1}{c \gamma} \operatorname{cosec} r_{3} \cos ^{2} \frac{\phi_{s}}{2}=0,
$$

or, since $\quad \cos r_{3} \cos r_{8} \sin ^{8} \frac{l}{2}=\sin r_{2} \sin r_{3} \cos ^{2} \frac{\phi_{1}}{2}$,

$$
\frac{1}{a \alpha} \sec r_{1} \sin ^{2} \frac{l}{2}+\frac{1}{b \beta} \sec r_{2} \sin ^{2} \frac{m}{2}+\frac{1}{c \gamma} \sec r_{3} \sin ^{3} \frac{n}{2}=0 .
$$

Its equivalent tangential equation is

$$
\sin \frac{l}{2} \sqrt{ }\left(p \sec r_{1}\right)+\sin \frac{m}{2} \sqrt{ }\left(p \sec r_{3}\right)+\sin \frac{n}{2} \sqrt{ }\left(r \sec r_{8}\right)=0 .
$$

Hence Dr. Casey's equation to a pair of circumscribed circles is derived by the theorem quoted in $\S 29$ :

$$
\sin \frac{l}{2} \sqrt{ }\left(S_{1} \sec r_{1}\right)+\sin \frac{m}{2} \sqrt{ }\left(S_{2} \sec r_{2}\right)+\sin \frac{n}{2} \sqrt{ }\left(S_{3} \sec r_{8}\right)=0 .
$$

Before I proceed to obtain this equation geometrically, the following lemmas are prefixed.

31. If two spherical circles be inverted from an arbitrary point, the product $\sin \frac{A B}{2}\left(\cot r_{2} \cot r_{9}\right)^{\prime}$ is unaltered, if $A B$ denote a common tangent arc. This expresses in another form that the angle, under which two small circles intersect, is unaltered by inversion. (\$24).

From $\$ 30$,

$$
\cos ^{2} \frac{\phi_{\mathrm{s}}}{2}=\cot r_{1} \cot r_{1} \sin ^{2} \frac{c}{2}
$$

32. If $A, B, C, D$ be four points on a spherical great or small circle,

$$
\sin \frac{B O}{2} \sin \frac{A D}{2}+\sin \frac{C A}{2} \sin \frac{B D}{2}+\sin \frac{A B}{2} \sin \frac{C D}{2}=0 .
$$

This extension of Ptolemy's theorem may be readily established, by joining the points to the centre, and expressing the semi-arcs in 
terms of the angles, which they subtend. Identically

$$
\sin \frac{a}{2} \sin \frac{b-c}{2}+\sin \frac{b}{2} \sin \frac{c-a}{2}+\sin \frac{c}{2} \sin \frac{a-b}{2}=0 .
$$

Let four circles whose radii are $r_{1}, r_{2}, r_{3}, r_{4}$ touch the preceding great circle $A B C D$ in the points $A, B, O, D$; then, if the system be inverted from any point, by $\S 31$,

$$
\sin \frac{B^{\prime} C^{\prime}}{2} \sin \frac{A^{\prime} D^{\prime}}{2}+\sin \frac{C^{\prime} A^{\prime}}{2} \sin \frac{B^{\prime} D^{\prime}}{2}+\sin \frac{A^{\prime} B^{\prime}}{2} \sin \frac{C^{\prime} D^{\prime}}{2}=0,
$$

a factor $\left(\cot r_{1} \cot r_{2} \cot r_{3} \cot r_{4}\right)^{1}$ being suppressed in each case.

In this case, the enveloping circle is small, and, in the tonching four circles, $A^{\prime} B^{\prime}, A^{\prime} C^{\prime}, \ldots .$. denote common tangent arcs to pairs of circles.

33. Lastly, let one of the four circles become a point-circle, as at $D^{\prime}$; then $A D^{\prime}, B D^{\prime}, C D^{\prime}$ denote tangent arcs drawn from a current point in the enveloping circle to the other three. Call these $t_{1}, t_{2}, t_{s}$.

Since $S_{1}, S_{2}, S_{8}$, of $\S 27$, denote the equations to the triad, for an external point,

$$
S_{1}=\cos r_{1}-\cos r_{1} \cos t_{1}=2 \cos r_{1} \sin ^{2} \frac{t_{1}}{2}
$$

and $S_{2}, S_{3}$ have like values. Thus, from the theorem

$$
\sin \frac{B^{\prime} C^{\prime}}{2} \sin \frac{t_{1}}{2}+\sin \frac{C^{\prime} A^{\prime}}{2} \sin \frac{t_{2}}{2}+\sin \frac{A^{\prime} B^{\prime}}{2} \sin \frac{t_{8}}{2}=0,
$$

is deduced Dr. Casey's equation to a pair of enveloping circles,

$$
\sin \frac{l}{2} \sqrt{ }\left(S_{1} \sec r_{1}\right)+\sin \frac{m}{2} \sqrt{ }\left(S_{2} \sec r_{2}\right)+\sin \frac{n}{2} \sqrt{ }\left(S_{3} \sec r_{3}\right)=0 .
$$

Cor. As has been shown in $\S 30$, this equation may take the form

$$
\cos \frac{\phi_{1}}{2} \sqrt{ }\left(S_{1} \operatorname{cosec} r_{1}\right)+\cos \frac{\phi_{2}}{2} \sqrt{ }\left(S_{2} \operatorname{cosec} r_{2}\right)+\cos \frac{\phi_{3}}{2} \sqrt{ }\left(S_{3} \operatorname{cosec} r_{3}\right)=0 \text {. }
$$

34. To deduce the equations to the inscribed and escribed circles in the triangle of reference and its associated triangles.

In these cases, $\pm S_{1}$ becomes

$$
a a+b \beta \cos c+c \gamma \cos b
$$

it denotes the side $B^{\prime} C^{\prime}$ of the polar triangle $A^{\prime} B^{\prime} C^{\prime}$, and is expressed as one of its coordinates $\left(a^{\prime}=0\right)$, since

$$
\begin{gathered}
6 V \boldsymbol{a}^{\prime}=a \alpha+b \beta \cos c+c \gamma \cos b ; \\
2 \mathrm{D} 2
\end{gathered}
$$


$\phi_{1}, \phi_{2}, \phi_{3}$ become the angles of $A^{\prime} B^{\prime} O^{\prime}$, and the required equations are

$$
\cos \frac{A^{\prime}}{2} \sqrt{ }\left( \pm \alpha^{\prime}\right)+\cos \frac{B^{\prime}}{2} \sqrt{ }\left( \pm \beta^{\prime}\right)+\cos \frac{C^{\prime}}{2} \sqrt{ }\left( \pm \gamma^{\prime}\right)=0 .
$$

35. To. deduce the equation to the circle described about the triangle of reference.

By the theorem of $\S 32$,

$$
\sin \frac{B C}{2} \sin \frac{A D}{2}+\sin \frac{O A}{2} \sin \frac{B D}{2}+\sin \frac{A B}{2} \sin \frac{C D}{2}=0,
$$

this is the vector equation to the circumscribed circle.

But, by Todhunter's Spher. Trig., p. 63,

$$
\tan R \sin B O \sin C D \sin B O D=4 \sin \frac{B D}{2} \sin \frac{C D}{2} \sin \frac{B C}{2},
$$

and

$$
\sin C D \sin B C D=\sin \alpha .
$$

By substituting in the preceding theorem, we derive the required equation $\frac{1}{\sin \alpha} \tan \frac{B O}{2}+\frac{1}{\sin \beta} \tan \frac{C A}{2}+\frac{1}{\sin \gamma} \tan \frac{A B}{2}=0$.

The vector-form of the equation to the circamscribed circle,

$$
\sin \frac{a}{2} \sin \frac{\rho_{1}}{2}+\sin \frac{b}{2} \sin \frac{\rho_{2}}{2}+\sin \frac{c}{2} \sin \frac{\rho_{3}}{2}=0,
$$

may be also thus reduced to the usual three-point equation. The vector-form may be expanded to

$$
\sin ^{2} A \cos ^{2}(S-A)\left(1-\cos \rho_{1}\right)^{2}+\ldots
$$

$\ldots-2 \sin B \sin O \cos (S-B) \cos (S-O)\left(1-\cos \rho_{2}\right)\left(1-\cos \rho_{8}\right)-\ldots=0$,

since

$$
\sin ^{2} \frac{a}{2} \sin B \sin C=-\cos S \cos (S-A),
$$

and

$$
6 V \cos \rho_{1}=a a+b \beta \cos c+c \gamma \cos b .
$$

This may be reduced to the complete square of the circle $\sin A \sin (S-A) \cos \rho_{1}+\sin B \sin (S-B) \cos \rho_{2}$

$$
+\sin C \sin (S-C) \cos \rho_{s}+\frac{\alpha}{2}(6 V)^{2} \sec S\left(\frac{\sin A}{\sin a}\right)^{2}=0 .
$$

Again, we find that

$$
\begin{gathered}
\sin A \sin (S-A)+\cos c \sin B \sin (S-B)+\cos b \sin C \sin (S-C) \\
=6 \nabla \frac{\sin A}{\sin a} \cot R=-\frac{1}{2} \sec S(\sin a \sin B \sin O)^{2}
\end{gathered}
$$


since the coordinates of the pole are

$$
\alpha \operatorname{cosec}(S-A)=\beta \operatorname{cosec}(S-D)=\gamma \operatorname{cosec}\left(S-C^{\prime}\right)=\sin R .
$$

This vector-form may be thus further reduced to the known forms

$$
a \alpha+b \beta+c \gamma=6 V
$$

and

$$
\frac{1}{a} \tan \frac{a}{2}+\frac{1}{\beta} \tan \frac{b}{2}+\frac{1}{\gamma} \tan \frac{c}{2}=0 .
$$

It may be remarked that this is the first and almost the sole application to Spherics, by Gudermann, of the three-point system, or, as he calls it, Plücker's system of coordinates, then (1830) recently introduced. (Sphärik, p. 160.)

For an associated triangle the vector equation of a circumscribed circle is $\quad \sin \frac{a}{2} \cos \frac{r_{1}}{2}+\cos \frac{b}{2} \sin \frac{r_{3}}{2}+\cos \frac{c}{2} \sin \frac{r_{3}}{2}=0$.

This may be reduced to the three-point equations

$$
-a \alpha+b \beta+c \gamma=6 V
$$

and

$$
-\frac{1}{a} \tan \frac{a}{2}+\frac{1}{\beta} \cot \frac{b}{2}+\frac{1}{\gamma} \cot \frac{c}{2}=0 .
$$

The limiting cases of the problem of contacts have thas been considered, when the radii become quadrants or zero.

36. Conversely, the equation to a pair of circles circumscribed about three others may be deduced from the equation to the circle inscribed in a triangle of reference.

The Fig. 5 is taken from Dr. Casey's memoir on the problem of contacts in planimetry (Proc: of Royal Irish Academy, Vol. Ix.). But the circles must be considered as small spherical circles, and the sides of $A B C$ as great circles. Three circles are drawn at the points of contact, of radii $r_{1}$, $r_{2}, r_{8} . \quad P$ is a point in the in-

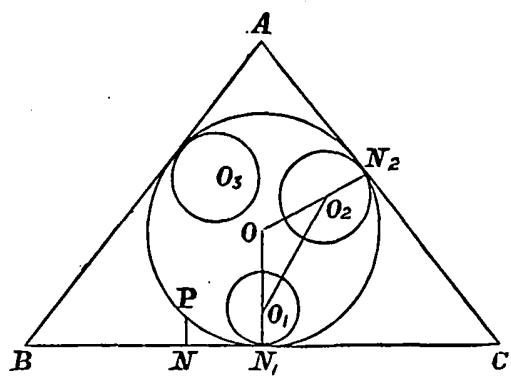

Fra. 5. scribed circle, radius $R$. 
If $S_{1}, S_{3}, S_{3}$ denote, as in $\S 27$, these three circles; then, if the coordinates be those of an external point $P$,

$$
\begin{aligned}
& S_{1}=\cos r_{1}-\cos r_{1} \cos t_{1}=\cos r_{1}-\cos P O_{1} \\
& =\cos r_{1}-\cos R \cos \left(R-r_{1}\right)-\sin R \sin \left(R-r_{1}\right) \cos P O O_{1} \\
& =2 \sin R \sin \left(R-r_{1}\right) \sin ^{2} \frac{1}{2}\left(P O O_{1}\right)=2 \operatorname{cosec} R \sin \left(R-r_{1}\right) \sin ^{2} \frac{1}{2}\left(P N_{1}\right) .
\end{aligned}
$$

Hence $\sin a=\sin P N_{1} \cos P N_{1} O=2 \sin ^{2} \frac{1}{2}\left(P N_{1}\right) \cot R$

$$
=S_{1} \cos R \operatorname{cosec}\left(R-r_{1}\right) \text {. }
$$

Again, $\quad \cos O_{1} O_{2}=\sin r_{1} \sin r_{3}+\cos r_{1} \cos r_{8} \cos n$

$$
=\cos \left(r_{1}-r_{9}\right)-2 \cos r_{1} \cos r_{2} \sin ^{2} \frac{n}{2},
$$

if, as in $\S 28, l, m, n$ denote common tangents to pairs of circles. Also

$$
\begin{aligned}
& \cos O_{1} O_{8}=\cos \left(R-r_{1}\right) \cos \left(R-r_{2}\right)+\sin \left(R-r_{1}\right) \sin \left(R-r_{8}\right) \cos \left(O_{2} O O_{8}\right) \\
& =\cos \left(r_{1}-r_{8}\right)-2 \sin \left(R-r_{1}\right) \sin \left(R-r_{2}\right) \sin ^{2} \frac{1}{2}\left(O_{1} O O_{8}\right), \\
& \text { and } \quad \cos \frac{O}{2}=\cos R \sin \frac{1}{2}\left(O_{1} O O_{8}\right) .
\end{aligned}
$$

Therefore

$$
\begin{aligned}
\cos ^{2} \frac{\sigma}{2} & =\sin ^{2} \frac{n}{2} \cos ^{2} R \cos r_{1} \cos r_{9} \operatorname{cosec}\left(R-r_{1}\right) \operatorname{cosec}\left(R-r_{2}\right) \\
& =\frac{1}{S_{1} S_{2}} \sin \alpha \sin \beta \sin ^{2} \frac{n}{2} \cos r_{1} \cos r_{2} .
\end{aligned}
$$

Substitate for $\sin a, \sin \beta, \sin \gamma$, in the equation to the inscribed circle,

$$
\cos \frac{A}{2} \sqrt{ }(\sin a)+\cos \frac{B}{2} \sqrt{ }(\sin \beta)+\cos \frac{C}{2} \sqrt{ }(\sin \gamma)=0 .
$$

The equation is deduced to a pair of circles circumscribed about three others,

$$
\sin \frac{l}{2} \sqrt{ }\left(S_{1} \sec r_{1}\right)+\sin \frac{m}{2} \sqrt{ }\left(S_{8} \sec r_{8}\right)+\sin \frac{n}{2} \sqrt{ }\left(S_{8} \sec r_{s}\right)=0 .
$$

37. The dirigent conic may be determined geometrically both in Spherics and in Planimetry.

The polar of a radical centre of three given circles with respect to 
any one of them intersects their axis of similitude in a point, whose connector with the centre of that circle touches the dirigent conic in that point.

On referring to $\S 27$, a radical centre is thus defined :-

$$
\begin{aligned}
(a \alpha+b \beta \cos c+c \gamma \cos b) \sec r_{1} & =(a \alpha \cos c+b \beta+c \gamma \cos a) \sec r_{3} \\
& =(a a \cos b+b \beta \cos a+c \gamma) \sec r_{3} .
\end{aligned}
$$

The polar of this point $(f, g, h)$ with respect to the circle $S_{1}$ is

$$
\begin{aligned}
&(a a+b \beta \cos c+c \gamma \cos b)(a f+b g \cos c+c h \cos b) \sec ^{2} r_{1} \\
&=a a(a f+b g \cos c+c h \cos b)+b \beta(a f \cos c+b g+c h \cos a) \\
&+c \gamma(a f \cos b+b g \cos a+c h),
\end{aligned}
$$

or $(a a+b \beta \cos c+c \gamma \cos b) \sec r_{1}=a a \cos r_{1}+b, b \cos r_{8}+c \gamma \cos r_{8}$.

This may be simplified to the form ( $(30)$

$$
a a \sin r_{1}-b \beta \sin r_{2} \cos \phi_{8}-c \gamma \sin r_{3} \cos \phi_{2}=0 .
$$

Through its intersection with the axis of similitude

$$
a a \sin r_{1}+b \beta \sin r_{2}+c \gamma \sin r_{3}=0,
$$

the $\operatorname{lin} \theta$

$$
b \beta \sin r_{8} \sec ^{2} \frac{\phi_{2}}{2}+c \gamma \sin r_{8} \sec ^{2} \frac{\phi_{3}}{2}=0
$$

passes, which is the tangent to the dirigent conic ( $\$ 30)$

$$
\begin{aligned}
& \frac{1}{a a} \cos ^{2} \frac{\phi_{1}}{2} \operatorname{cosec} r_{1}+\frac{1}{b \beta} \cos ^{2} \frac{\phi_{2}}{2} \operatorname{cosec} r_{2}+\frac{1}{c \gamma} \cos ^{2} \frac{\phi_{8}}{2} \operatorname{cosec} r_{3}=0, \\
& \text { or } \quad \frac{1}{a a} \sin ^{2} \frac{l}{2} \sec r_{1}+\frac{1}{b \beta} \sin ^{2} \frac{m}{2} \sec r_{2}+\frac{1}{c \gamma} \sin ^{2} \frac{n}{2} \sec r_{3}=0 .
\end{aligned}
$$

Three points $A, B, C$ are therefore known, and the tangents at those points, of the dirigent conic; three other points can be found from the property given in $\S 5$,

$$
\cot \rho_{1}+\cot \rho_{8}=2 \cot \rho .
$$

And Pascal's theorem enables us to construct the conic, when five of its points are given.

38. Another solution of the problem of contacts is suggested by the perfect duality which exists in Spherical Geometry. 
The dual forms of the equations (1) of $\S 27$ denote in the tangential form, when they are referred to the polar triangle as the triangle of reference, three circles, which are polar or complementary to the three given circles $S, S_{2}, S_{3}$.

All three complementary circles have the type

$$
a p-b q \cos C-c r \cos B=6 V \sin r_{1} \text {. }
$$

An anxiliary Jacobian, the dual of the Jacobian in $\S 27$,

$$
a p \sin r_{1}+b q \sin r_{2}+c r \sin r_{3}=6 \mathrm{~V},
$$

denotes a circle whose common tangents with these complementary circles are quadrants in length.

A pair of circles, which shall be circumscribed about these three circles, will be contangential with their Jacobian,-their common centre of similitude being a radical centre of the given circles.

The rest of the analytical solution would proceed as in $\S 27$, nutatis mutandis.

39. The axes of similitude of the given circles $S_{1}, S_{2}, S_{3}$ in $\S 27$ are quadrantal polars of the radical centres of their complementary circles in $\$ 38$.

This is evident both geometrically and analytically.

The line-equations of the primitive circles $S_{1}, S_{2}, S_{3}$ are

$$
p^{2}=\sin ^{2} r_{1}, \quad q^{2}=\sin ^{2} r_{2}, \quad r^{2}=\sin ^{2} r_{3} .
$$

When combined, they denote the four axes of similitude. The threepoint equivalent equations to their dual circles of $\S 38$ are

$$
\alpha^{2}=\cos ^{2} r_{1}^{\prime}, \quad \beta^{\prime 2}=\cos ^{2} r_{2}^{\prime}, \quad \gamma^{\prime 2}=\cos ^{2} r_{3}^{\prime} \text {. }
$$

When combined, they denote the four radical centres. The accents are added, to indicate reference to the polar triangle.

It is evident from a diagram that $p=\alpha^{\prime}, q=\beta^{\prime}, r=\gamma^{\prime}$; and the radii of the triads of circles are complementary to each other.

40. The theorem here used may be generalised. Every tangential equation to a spherical curve referred to a triangle $A B C$ has a corresponding three-point equation of the same form, referred to the polar triangle $A^{\prime} B^{\prime} O^{\prime}$, to denote its complementary or polar curve.

[41. Note on $\S \S 23,24$.- -In plano a circle is only dually inverted into another circle, when the angles of inversion are complementary.

$$
\tan \frac{\rho_{1}}{2} \tan \frac{\rho_{4}}{2}=\tan \frac{\rho_{3}}{2} \tan \frac{\rho_{3}}{2}=1, \text { and } R=\frac{\pi}{2} .
$$


Then the Boothian circle $\frac{c}{p}=1+m \xi+n \eta$ ( $\xi$ being the common radical axis) is transformed into $\frac{c}{p}=-1-m \xi \pm n \eta$, and the common tangent of two circles_is analtered in length by Dual Inversion.]

Reciprocation in Statics. By Prof. Genest, M.A.

[Read June 10th, 1886.]

If a system of complanar forces $P_{1}, P_{2}$, \&c., be in equilibrium, and any transversal meet their lines of action in $I_{1}, I_{2}$, \&c., at angles $\theta_{1}, \theta_{9}$, \&c. (estimated with due regard to the directions of the forces and one direction of the transversal), then, resolving perpendicularly to the transversal, we have a system of parallel forces $P_{1} \sin \theta_{1}, P_{2} \sin \theta_{9}$, \&c., at $I_{1}, I_{2}$, \&c., in equilibrium.

The opposite to one of the above forces is defined as the resultant of the others. The cases of four and five forces led to the theorems in the right-hand column of the following

Contrast.

1. If $I$ be the centre of parallel forces $l, n, n$ at the points $A, B$, $O$, then forces $l I A, m I B, n I C$ are in equilibrium. (Leibnitz.)
1. If $\delta$ be the line of action of the resultant of forces $l, m, n$, acting along the sides $a, b, c$, then parallel forces $l \sin (\delta a), m \sin (\delta b)$, $n \sin (\delta c)$ are in equilibriam.
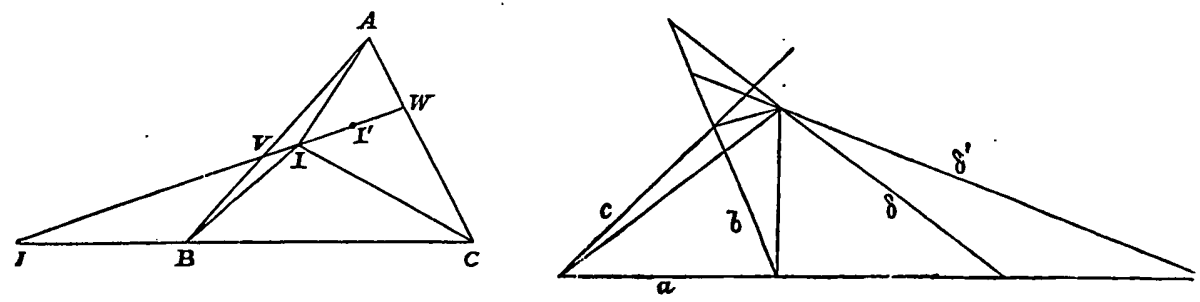